\title{
In-situ Phase Transformation and Deformation of Iron at High Pressure and Temperature
}

\author{
Lowell Miyagi ${ }^{1}$, Martin Kunz ${ }^{2}$, Jason Knight ${ }^{2}$, James Nasiatka ${ }^{2}$, Marco Voltolini ${ }^{1}$, and Hans-Rudolf \\ Wenk ${ }^{1}$ \\ ${ }^{1}$ Department of Earth and Planetary Science, University of California, Berkeley, CA 94720 \\ ${ }^{2}$ Advanced Light Source, Lawrence Berkeley Laboratory, Berkeley, CA 94720.
}

\begin{abstract}
With a membrane based mechanism to allow for pressure change of a sample in a radial diffraction diamond anvil cell (rDAC) and simultaneous infra-red laser heating, it is now possible to investigate texture changes during deformation and phase transformations over a wide range of temperature-pressure conditions. The device is used to study bcc $(\alpha)$, fcc $(\gamma)$ and hcp $(\varepsilon)$ iron. In bcc iron, room temperature compression generates a texture characterized by (100) and (111) poles parallel to the compression direction. During the deformation induced phase transformation to hep iron, a subset of orientations are favored to transform to the hcp structure first and generate a texture of (01-10) at high angles to the compression direction. Upon further deformation, the remaining grains transform, resulting in a texture that obeys the Burgers relationship of $(110)_{\mathrm{bcc}} / /(0001)_{\mathrm{hcp}}$. This is in contrast to high temperature results that indicate that texture is developed through dominant pyramidal $\langle\mathrm{a}+\mathrm{c}\rangle\{2-1-12\}\langle 2-1-13\rangle$ and basal $(0001)\langle 2-1-10\rangle$ slip based on polycrystal plasticity modeling. We also observe that the high temperature fcc phase develops a 110 texture typical for fcc metals deformed in compression.
\end{abstract}

\section{Introduction}

A technique that has emerged in recent years to study the rheology and elasticity of materials in-situ at ultra-high pressures is the use of the diamond anvil cell (DAC) in radial $\mathrm{x}$-ray diffraction geometry (rDAC). ${ }^{1-4}$ With this technique diamonds are used to impose stress and pressure on a material to induce elastic and plastic deformation. An xray beam is brought in orthogonal to the compression axis through $\mathrm{x}$-ray transparent gasketing materials and deformation effects are observed on diffraction images as 
intensity changes along Debye rings (texture) and variations in d-spacings relative to the compression direction (lattice strain).

While previous work using the rDAC has provided important information on insitu rheological behavior through interpretation of texture patterns, these studies were limited to ambient temperature. As a result little is known about the behavior of materials at high-temperature and ultra-high pressures. This is of particular importance to the field of Earth and planetary sciences as it is questionable whether room temperature studies are appropriate for extrapolation to behavior in planetary interiors where materials are deforming both at high-pressure and temperature. In order to expand rDAC experiments to the high-temperature regime, an in-situ laser-heating system for radial diffraction geometry has been developed at the Advanced Light Source (ALS) of the Lawrence Berkeley Laboratory (LBL). ${ }^{5}$ Previously, pressure/stress increase in rDACs has been controlled through load screws pushing the piston of the rDAC into its cylinder. This requires manual intervention and is incompatible with in-situ observations of the effects of pressure and stress increase at high-temperature. Furthermore, manual pressure change requires re-aligning the sample on the x-ray beam, which is time intensive and thus limits the resolution in pressure steps for radial diffraction experiments, even at ambient temperatures. A remote controlled pressure changing mechanism is thus required. A membrane driven DAC for axial diffraction has been developed, but the closed design in the radial direction does not allow its application to radial diffraction. ${ }^{6}$ Alternately a motor driven mechanism can be used to remotely change pressure on a screw-driven Mao-Bell DAC from outside a synchrotron experimental hutch. ${ }^{7}$ While the solution of a remotely controlled mechanical cell is advantageous in terms of long-term pressure stability, it bears some drawbacks in terms of weight and flexibility, especially in view of the need to combine it with in-situ laser heating. Here we present an approach based on gas-membrane technology. Instead of designing a new rDAC, we aimed to construct a frame applicable to various designs of rDACs. In our design, we paid special attention to flexibility (easy to accommodate several types of rDAC designs), weight (it should not exceed the load limitations of the high-precision stages on the end-station) and open access (for combination with in-situ 
laser heating). This development enables the exploration of deformation mechanisms simultaneously at high-temperature and ultra high-pressure.

Iron is a good candidate for pilot experiments with our new deformation system, as it has been well studied due to its importance to engineering and deep Earth geophysics. The pressure temperature phase diagram of iron is shown in Figure $1 .{ }^{8} \mathrm{At}$ ambient conditions iron takes a bcc $(\alpha)$ structure, and upon heating transforms to the fcc structured $\gamma$ phase (Figure 1). This transformation in steel is of critical importance in engineering because there is a significant reduction in material strength associated with the transformation. The high pressure $\varepsilon$ phase has an hcp structure, and at ambient temperature this transformation occurs at pressures above $11 \mathrm{GPa}^{9,10}$ Under hydrostatic conditions the phase boundary is sharp but under non-hydrostatic conditions becomes spread over a larger pressure range. ${ }^{11}$ Heating the hep phase induces a transformation to the fcc phase ${ }^{12-14}$ (Figure 1).

It is likely that the bulk of the Earth's solid inner core is composed of hcp iron (ع). ${ }^{15,16}$ After the discovery of seismic anisotropy in the inner core, ${ }^{17,18}$ it was proposed that origin of this anisotropy may be due to the development of preferred orientation of crystals in the inner core. ${ }^{19-23}$ The texture evolution of hep Fe was studied at pressures up to $220 \mathrm{GPa}$ at ambient temperature using the rDAC. Based on the texture pattern with a maximum of 0001 poles near the compression axis, it was inferred, through the use of polycrystal plasticity modeling, that slip on the basal (0001) plane was dominant under these conditions. ${ }^{24-26}$ In contrast to these experiments, dislocations consistent with pyramidal $\langle\mathrm{a}+\mathrm{c}\rangle$ slip on the $\{2-1-12\}\langle 2-1-13\rangle$ system were observed in an hcp $\mathrm{Ni}-\mathrm{Cr}$ stainless steel analog using transmission election microscopy (TEM). ${ }^{27}$ In rDAC experiments it was observed that in compression the $\alpha$-phase of Fe developed two fiber components of (100) and (111), ${ }^{25}$ in agreement with textures observed by metallurgists. ${ }^{28,29}$ During the phase transformation of the bcc to the hcp phase, hep Fe inherited a texture from the parent bcc phase consistent with the Burgers orientation relationship. ${ }^{25,30}$

In order to follow up on these previous room temperature experiments we use our new device to deform polycrystalline iron at high pressure and temperature and obtain insitu radial diffraction images. In a first run we deform Fe to high-pressure at ambient 
temperature in order to convert bec Fe to hep Fe and then decompress back to the bcc phase (Figure 1 path A). This allows us to evaluate the capabilities of our membrane loading system on a material that has been well documented with radial diffraction. In a second run we combine laser heating with our membrane loading system to first deform the bcc phase then convert it by heating into the fcc phase. Finally the fcc phase is converted to hcp by increasing pressure followed by deformation of the hcp phase at simultaneous high pressure and temperature (Figure 1 path B). Emphasis of this study is on texture changes, revealed by diffraction images and interpretation of those changes in terms of orientation variant selection during phase transformations and active deformation mechanisms.

\section{Experimental Technique}

X-ray diffraction experiments were performed on the dedicated high-pressure beamline 12.2.2 at ALS. $^{31,32}$ A setup for single-sided in-situ laser heating of a DAC in radial diffraction geometry was recently implemented on this beamline. ${ }^{5}$ In order to remotely change pressure, we constructed an rDAC holding frame which is compatible with various radial diffraction cells. The device consists of a frame-body, housing the rDAC in use, a membrane, and a base plate to constrain the piston against the cylinder (Figure 2). Spacer blocks allow the holding frame to accommodate various rDAC designs within the same holding frame such as the Mao style panoramic rDAC and the smaller Merkel style rDAC (Figure 3). The whole assembly is constructed of Prodec stainless steel. To minimize restrictions in x-ray access to the sample, the holding frame has two large windows along the x-ray axis (Fig. 2, 3). The side-posts of the frame provide sufficient mechanical stability for an expected tensile force of $40 \mathrm{kN}$ as established by finite element analysis. This should be sufficient to withhold a membrane pressure in excess of 200 bars. The cap of the holding frame has a smaller access window to allow for imaging the sample and focusing an infra-red laser onto it (Figure 2, 3). The device was built by Phillips Machining in Martinez, CA.

The loaded rDAC is placed in an upright position (i.e. load axis vertical) into the holding frame with its load-screws removed. This geometry not only allows easy laser access but additionally allows the user to rotate the cell about the compression axis 
during x-ray exposure in order to improve grain statistics while the laser is in operation. The gas-driven membrane is sandwiched between the base plate and the piston of the rDAC with the base plate mechanically coupled to the holding frame. The membrane is connected to a pressure reducer with a 1/16 inch pressure tube through the base-plate. Pressure change is achieved by applying Helium gas pressure ( $0-\sim 150$ bars $)$ onto the membrane which in turn pushes the piston into the cylinder of the rDAC. Custom designed membranes were produced by a local company (DVM, Livermore, CA). In order for the membrane to fit into the base of the holding-frame, the pressure tube had to be welded on to it such that it exits the membrane parallel to its plane with a minimal vertical displacement. Furthermore, a more solid welding rim was developed in order to improve the reliability of the gas-membranes. Maximal stroke of the membrane is $\sim 0.5$ $\mathrm{mm}$.

For the room temperature deformation run the sample of Alfa Aesar Fe powder of $99.9+\%$ purity and spherical grains of $<10 \mu \mathrm{m}$ was loaded into a 2-stage kapton/boronepoxy gasket ${ }^{33}$ with an $80 \mu \mathrm{m}$ sample chamber. No pressure medium was added to maximize the macroscopic deviatoric stress. For the second run at high-temperature a sandwich type sample assembly was used. In order to reduce temperature gradients in the sample, the polycrystalline Fe sample was loaded into a $50 \mu \mathrm{m}$ sample chamber. A small Pt flake, Alfa Aesar foil, $0.004 \mathrm{~mm}$ thickness and 99.95\% purity was imbedded in the Fe as an internal pressure standard, and each side of the sample was capped with platelets of polycrystalline $\mathrm{MgO}$, Alfa Aesar $99.9+\%$ purity to serve as insulating material between the diamonds and the sample.

The cell within the holding frame is placed onto the goniometer and aligned on its rotation axis, which serves as a reference point for the detector to sample distance. ${ }^{31}$ Sample to detector distance and detector non-orthognality were calibrated using a $\mathrm{LaB}_{6}$ standard. For run 1 at ambient temperature no pressure calibrant was used and pressure was calibrated with a $3^{\text {rd }}$ order Birch-Murnaghan equation of state for bcc Fe and hep $\mathrm{Fe}^{34,35}$ For the high temperature run, pressures were calibrated with the equation of state for $\mathrm{Pt}^{36}$ Laser heating was performed applying a fiber IR laser and optical set-up for radial diffraction. ${ }^{5}$ Temperature was measured by fitting its spectral glow to a Planck function. ${ }^{32}$ 
In the first run (A) pressure was increased at ambient temperature in $\sim 0.25 \mathrm{GPa}$ increments taking in-situ diffraction images at each step up to a pressure of $\sim 22 \mathrm{GPa}$ at which point the sample was fully converted to the hcp phase. Once full conversion was achieved the sample was incrementally decompressed back to the bcc phase to $\sim 4 \mathrm{GPa}$. For the high-temperature deformation cycle (B) the sample was first compressed to $\sim 10$ $\mathrm{GPa}$ at ambient temperature at which point the laser was turned on and power was incrementally increased up to $15 \mathrm{~W}(1900 \pm 150 \mathrm{~K})$, taking in-situ diffraction images at each power increase. Once a power of $15 \mathrm{~W}$ was reached, pressure was increased incrementally at high-temperature in 1-2 GPa steps up to $30 \mathrm{GPa}$, recording in-situ diffraction patterns during pressure increase (Figure 1). During data collection the sample was oscillated $\pm 5^{\circ}$ about the loading axis to increase grain statistics. Once a pressure of $\sim 30 \mathrm{GPa}$ was attained, the laser was turned of and the sample was decompressed at room temperature. No data was collected during room temperature decompression.

Radial diffraction images were quantitatively analyzed for texture using the Rietveld method as implemented in the software package MAUD. ${ }^{37}$ Rietveld refinement with MAUD accounts for instrumental parameters such as beam center, detector tilt, peak shape, backgrounds, and azimuthal absorption differences. Once instrument parameters have been fit, structural and microstructural parameters including phase proportions, differential stresses and texture were refined. For refinements an angular $2 \theta$ range of $12^{\circ}-25.5^{\circ}$ at $25 \mathrm{KeV}$ was used for the room temperature run, and a range of $10^{\circ}-22^{\circ}$ at 30 $\mathrm{KeV}$ was used for the high temperature run. Textures were calculated using the tomographic E-WIMV algorithm, which is similar to the WIMV model ${ }^{38}$ but allows for arbitrary orientations. For texture calculation cylindrical symmetry was imposed around the compression axis. The resolution of orientation space was $15^{\circ}$ and the refined orientation distribution was exported to BEARTEX ${ }^{39}$ and further smoothed with a $10^{\circ}$ Gauss filter. In the case of axial compression, textures can be compactly represented with an inverse pole figure (IPF). An IPF shows the orientation of the compression axis relative to the crystallographic directions. The asymmetric sector of the IPF conforms with the crystal symmetry. Pole densities are expressed in multiples of random 
distribution (m.r.d.), where 1 m.r.d. corresponds to a random distribution and in the case of a single crystal the m.r.d. value would be equal to infinity.

\section{Results and Discussion}

\section{III-I. Room Temperature Deformation}

Results for the room temperature compression cycle of Fe are shown in Figures 4, 5, and Table SM-I. Figure 4 shows pressure versus run number, and texture results are shown with selected inverse pole figures. Of the 209 diffraction patterns collected during room temperature compression and decompression, full analysis was performed on 29 patterns and selected IPFs are shown in Figure 4. Details about pressure, lattice parameters, phase proportions and texture strength for all analyzed patterns are summarized in Table SM-I.

During the room temperature run we are able to generate a homogeneous pressure increase yielding a smooth compression-decompression curve (Figure 4). This indicates that our membrane loading frame has the capability for finely tuned pressure/stress increase and decrease and will allow us to move closer to the goal of controlled strain rates in the rDAC. Additionally, the design of an external loading frame with various spacers is very convenient as it allows the use of several different $\mathrm{rDAC}$ designs to perform in-situ experiments. The compact design of the device makes it possible to move it to different synchrotron facilities.

During compression, we observe the appearance of the high pressure hcp phase at $10 \mathrm{GPa}$ (Figure 4). Continued compression results in an increase in the proportion of the hcp phase at the cost of the bcc phase (Figure 5). During the phase transformation we noticed some discrepancies in the pressures obtained from bcc and hep phases, respectively. At $\sim 22 \mathrm{GPa}$ the sample is fully converted (Table SM-I, Figure 4, 5). The large pressure range over which the transformation occurs is consistent with previous experimental work ${ }^{11,25}$ and can be attributed to stored energy due to lattice strains that do not contribute to overall deformation but rather accommodate grain-grain compatibility. ${ }^{40}$

During decompression we do not observe the formation of the bcc phase until about $8 \mathrm{GPa}$ (Table SM-I, Figure 4, 5 \# A 172). With continued pressure decrease, we observe an increase in the proportion of the bcc phase coupled with a decrease in the 
proportion of the hcp phase (Figure 5). The hcp phase persists well into the stability field of the bec phase (Table SM-I, Fig. 4).

The pressure discrepancies observed during the phase transformation may be partly due to the different equations of state used; however this difference is likely to be small. It is known that the coexistence of multiple phases during a phase transformation can cause volume anomalies between the phases due to differences in compressibility and thermal expansion. ${ }^{41}$ Additionally anomalous unit cell parameters have been observed during the bcc-hcp transformation in iron. ${ }^{42}$ It is likely that the pressure differences observed are due to these effects. It is also possible that there are pressure gradients within the cell. It is interesting to note that during decompression, measured pressures for the two phases agree much better than during compression (Figure 4). This may indicate that differential stresses are lower during the phase transformation in decompression and as a result volume anomalies are smaller. Additionally, pressure gradients may be lower during decompression.

\section{III-II. Room Temperature Texture}

At the start of the experiment the IPF shows that the sample (bcc phase) has no significant preferred orientation (Figure 4, \# A 26( $\alpha)$ )). Upon commencement of compression, the bcc phase develops a texture characterized by maxima in the 100 and 111 directions and a minimum at 110 (Figure 4, \# A 69( $\alpha)$ ). This agrees with compression textures reported for bcc metals ${ }^{28,29}$ and is attributed to dominant slip on $\{110\}\langle 111\rangle$. This texture is also consistent with previous texture measurements on bcc Fe in the rDAC. ${ }^{25}$

During the initial stages of the phase transformation (Figure 4, \# A 67(ع)), the hcp phase develops a maximum in the 01-10 direction. As the phase transformation progresses, this texture becomes broader (Figure 4, \# A 72(E)), and shifts to form a maximum at 11-20 as the phase transformation is completed (Figure 4, \# A 125(ع)). Previous rDAC measurements on hcp Fe reported that after the phase transformation, hcp Fe developed a maximum at 11-20. This was interpreted as a transformation texture that was inherited from the parent bcc phase ${ }^{25}$ based on Burgers' orientation relationship ${ }^{30}$ $(110)_{\mathrm{bcc}} / /(0001)_{\mathrm{hcp}}$ and [-11-1 $]_{\mathrm{bcc}} / /[11-20]_{\mathrm{hcp}}$ (Figure 4 inset). An alternate and nearly 
equivalent description is the martensitic mechanism where $\{1-100\}\langle 11-20\rangle_{\mathrm{hcp}}$ becomes $\{1-12\}\langle-111\rangle_{\text {bcc. }}{ }^{43}$ Indeed we do observe in our IPFs that these corresponding orientations have qualitatively similar texture strengths. 100 and 111 have maxima in the bcc phase as does the corresponding hep orientation of 11-20. Additionally 110 is depleted in the bcc phase and likewise the 0001 hcp orientation is also depleted (Figure 4, \# A 69( $\alpha$ ), \# A 125(E)). The maximum that is initially observed at $01-10$ was not observed in previous measurements.

The bcc-hcp transformation has been extensively modeled ${ }^{40,44}$ and it is generally accepted that the presence of defects can enhance the phase transformation. ${ }^{45}$ In particular the bcc-hcp transition in $\mathrm{Zr}$ was modeled in the presence of edge dislocations and it was found that dislocations enhanced the rate of transformation. ${ }^{46,47}$ For $\{110\}\langle 001\rangle$ dislocations in the bcc phase, transformation to the hep phase first occurs in a region close to $45^{\circ}$ from the dislocation as this is the region with maximum shear strain. ${ }^{46}$

According to the orientation relationship for bcc-hcp phase transformations the $\langle 01-10\rangle_{\mathrm{hcp}}$ direction would correspond to $\langle 112\rangle_{\mathrm{bcc}}$ (Figure 4, inset, \# A 69( $\left.\alpha\right)$, \# A $67(\varepsilon)$ ). Those grains with $\{112\}$ perpendicular to the compression direction will have maximum compressive stress along $\langle 112\rangle_{\mathrm{bcc}}$. The $\langle 112\rangle_{\mathrm{bcc}}$ direction is near $45^{\circ}$ to the $\{110\}\langle 001\rangle$ dislocation and thus grains with this orientation would be most favorably oriented to develop this dislocation and to transform to the hep phase. As a result grains with $\{112\}$ perpendicular to compression will transform to the hep phase first, yielding a maximum

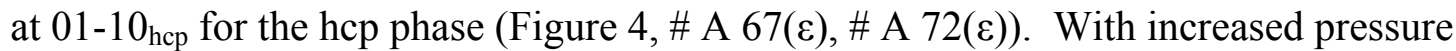
the hcp phase becomes more energetically favored and other orientations will transform eventually producing a texture in the hcp phase that corresponds to the texture of the parent bcc phase (Figure 4, \# A 69( $\alpha$ ) and \# A 125(ع)).

An alternate explanation for this behavior is analogous to recrystallization in $\mathrm{NaCl}^{48}$ In this model recrystallization favors plastically "soft" grains over plastically "hard" grains. During recrystallization the "hard" grains have higher strain energy and are less stable. The result is that "hard" grains are annihilated while "soft" orientations nucleate and grow. ${ }^{48}$ If plastically "soft" grains are more stable due to lower strain energy then they will be less likely to transform. This means that orientations at 100 and 111 in the bcc IPF will be the last grains to transform as they are the most stable (Figure 
4, \# A $69(\alpha)$ ). This will generate a minimum at the corresponding 11-20 orientation in the hcp phase (Figure 4 inset, \# A 67(ع)), as these orientations have yet to transform. Additionally 110 bcc orientations are depleted in the IPF (Figure 4 inset, \# A 69( $\alpha$ )), so there are very few orientations to transform to the hcp phase. This will generate a minimum at 0001 in the hep phase (Figure 4, \# A 67(ع)). Since the majority of the orientations that are favored to transform to the hcp phase are near $112_{\mathrm{bcc}}$ a maximum will form at the corresponding 01-10 hcp orientation (Figure 4, \# A 67(E)). As the hcp phase becomes more favored, eventually the remaining orientations near $100_{\mathrm{bcc}}$ and $111_{\text {bcc }}$ will also transform to the hep phase and generate the maximum that is observed at 11-20 hcp upon completion of the phase transformation (Figure 4, \# A 125(ع)).

As the bcc-hcp transformation occurs there are many symmetrically equivalent orientation variants that exist between the phases. The number of variants depends on the exact mechanism of the transformation. For the Burgers mechanism the bcc-hcp transformation has 12 equivalent variants. ${ }^{30}$ If all variants are equally favored, texture will become weaker through the phase transformation. However with variant selection the texture will remain strong after the phase transformation. ${ }^{49}$ We observe that the latter is the case, indicating that variant selection plays an important role during the bcc to hcp transformation. This is consistent with previous observations. ${ }^{25}$

Upon decompression, the hcp phase develops a 0001 maximum and the 11-20

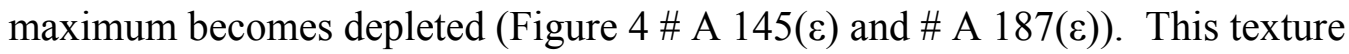
evolution was observed during compression in previous experiments. ${ }^{24-26}$ Although pressure is decreasing in our sample due to decompression, the deviatoric stress component will still be in a compressive stress state, and thus these results are not inconsistent with previous measurements. A texture with a 0001 maximum has been attributed to dominant basal $(0001)\langle 2-1-10\rangle$ and prismatic $\{10-10\}\langle-12-10\rangle$ slip. $^{24,25}$ During the phase transformation back to the bcc phase, the hcp phase becomes depleted

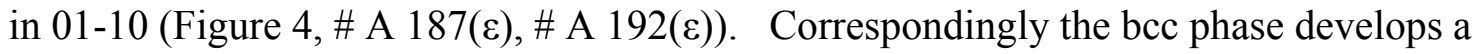
maximum at 112 (Figure 4, \# A 172( $\alpha$ )). This is consistent with the dislocation propagated phase transformation suggested for the compression phase of the experiment. Interestingly, as the transformation progresses to completion, the bcc phase becomes nearly random again. This indicates that for the reverse transformation (hcp to bcc Fe) 
variant selection is not as pronounced. It does however appear that those orientations with $\{112\}$ at high angle to the compression direction maintain texture during the back transformation and this subset of grains may exhibit variant selection.

\section{III-III. High Temperature Deformation}

The high-temperature cycle was very successful since it allows us for the first time to combine in-situ radial x-ray diffraction of a sample compressed to high pressure and heated with an infrared laser to high-temperatures. The described set-up allows change in both pressure and temperature while diffraction patterns are recorded. This enabled us to finely step the iron sample through its phase transitions in P-T space, by varying both pressure and temperature.

For the high temperature deformation cycle, deformation was performed more quickly to minimize the heating time necessary and only 35 diffraction images were collected. Of these, 15 images were selected for detailed analysis and results are shown in Table SM-II. In-situ radial diffraction images are shown in Figure 6 and relative phase proportions of the three Fe phases versus run number are shown in Figure 7. In Figure 6 images have been "unrolled" using the cake routine in FIT2D. ${ }^{50}$ Only one quadrant of each image is shown and compression and extension directions are indicated by arrows. At the start of the experiment, diffraction images show three phases, the platinum pressure marker, the $\mathrm{MgO}$ insulation, and the bcc Fe sample (Figure 6a). All lines are straight, exhibiting no significant lattice strain, as is expected. While bcc $\mathrm{Fe}$ and $\mathrm{MgO}$ exhibit no significant texturing, the Pt shows texture. The Pt used in this experiment is a foil and texturing is a result of processing. After compression to $10 \mathrm{GPa}$, unit cell parameters have decreased and we observe an azimuthal variation in peak position due to lattice strains. Diffraction lines have also developed systematic intensity variations that are indicative of texture (Figure $6 b$ ).

After the initial compression, temperature was incrementally increased to 1900 $( \pm 150) \mathrm{K}$ (Table SM-II). During the first heating period, we observe the formation of both the hcp and the fcc phases with slightly more of the hcp phase than the fcc phase (Table SM-II, Figure 6c, 7). Upon reaching the maximum temperature of $1900( \pm 150) \mathrm{K}$ the hcp and fcc phases increase in proportion and we observe a coupled decrease in the 
phase fraction of the bcc phase (Figure 7) as well as relaxation of lattice strains (Figure 6c). At these conditions there is more of the fcc phase than the hep phase (Table SM-II, Figure 7). We also observe grain growth and the development of a spotty pattern containing the $\mathrm{Pt}, \mathrm{MgO}$ and a second higher temperature fcc phase ( $\left.\gamma_{2}\right)$ (Figure 6c). The diffraction rings in these regions were too spotty for an accurate interpretation and were not analyzed except to extract unit cell parameters from the Pt for pressure calibration.

We observe significant temperature gradients due to the smaller size of the laser hotspot compared to the sample. Temperature measurements made during laser heating only correspond to temperatures at the center of the hotspot. Presumably the edges of the sample are considerably colder. This, in addition to probable pressure gradients, means that the $\mathrm{x}$-ray beam passes through the whole sample and thus records a considerable region of the Fe phase diagram (Figure 1). Temperature gradients can complicate pressure calibration as it is difficult to tell what part of the sample is at the measured temperature. However in this experiment the Pt flake was well centered and was visually confirmed to be in the laser hotspot. In addition, the Pt heavily recrystallized upon heating, indicating that it is in the very high temperature $(1900 \mathrm{~K})$ region of the sample.

As a result we assume the $\mathrm{Pt}$ is at the measured hotspot temperature and use this temperature to calibrate pressure at the center of the sample.

At a temperature of $1900 \mathrm{~K}$, pressure was increased again and we observe an increase of the hcp phase at the expense of both the bcc and fcc phases (Table SM-I, Figure 7). With further deformation and pressure increase at high temperature we see the development of lattice strains and an increase in texture strength in all phases (Figure 6d). The fcc phase has disappeared except for the most recrystallized region, and at the same time, the hcp phase has become stronger. Diffraction lines have become smoother due to grain size reduction associated with deformation (Figure 6d).

\section{III-IV High Temperature Texture}

For the high temperature cycle, the bcc phase initially has a random texture (Figure 7, \# B 115( $\alpha)$ ). During this initial compression at ambient temperature the bcc phase develops the typical bec compression texture of 100 and 111 maxima with a minimum at 110 . This texture is similar to that obtained in the room temperature cycle. 
This texture does not change much with increased temperature or continued deformation (Figure 7, \# B 139( $\alpha)$ ).

After transformation the fcc phase develops a maximum at 110 and minima at 100 and 111. Transformation textures for the bcc to fcc transformation in steel has been extensively studied. ${ }^{51}$ The textures observed here are consistent with a phase transformation following the Kurdjumov and Sachs orientation relationship ${ }^{52}$ in which $\{111\}_{\text {fcc }} / /\{1-10\}_{\text {bcc }}$ and $\langle 1-10\rangle_{\text {fcc }} / /\langle 111\rangle_{\text {bcc }}$ (Figure 7, inset). In this case the 100 and 111 maxima in the bcc phase correspond to the 110 maximum for the fcc phase (Figure 7, \# B $139(\alpha)$ and \# 133( $\gamma)$ ). The presence of strong texture through the phase transformation indicates that variant selection is active for the bcc Fe to fcc Fe phase transformation, as has been previously observed in ultra-low carbon steel. ${ }^{53}$ The 110 maximum in the fcc phase remains the same with continued heating and deformation. This texture can also be generated by slip on $\{111\}\langle 110\rangle$ and is the typical compression texture in fcc metals. ${ }^{29}$ It has also been observed in rDAC deformation experiments on $\mathrm{fcc} \mathrm{Cu}^{54}$

During temperature increase the hcp phase appears and has a texture with a 11-20 maximum (Figure 7, \# B 128(e)). These textures indicate that variant selection is also important in the bcc Fe to hcp Fe transformation at high temperature. We do not observe the initial development of the 01-10 maximum that was observed in the room temperature run. This may be due to the different transformation path. In the room temperature run the transformation was stress induced where as in the high temperature run the hcp phase appears during heating and presumably the phase transformation is thermally induced. Additionally transformation kinetics are likely to be very different at room temperature and high temperature. With continued deformation and heating the maximum at 11-20 disappears and the texture becomes weaker. We also see the formation of a weak maximum at 01-10 and a girdle $30^{\circ}$ offset from 0001 (Figure 7, \# B 138(ع)).

Recrystallization experiments on hep Ti alloys showed that during recrystallization the 11-20 orientation switches to $01-10 .{ }^{55}$ This would explain the depletion of the 11-20 maximum and the formation of a 01-10 maximum. However, the girdle $30^{\circ}$ offset from 0001 cannot be explained by this and is likely due to slip. As compression continues we observe that the girdle $30^{\circ}$ from 0001 remains but the 11-20 maximum appears again (Figure 7, \# B 141(ع)), becoming stronger and eventually 
spreading to form a girdle around the periphery of the IPF (Figure 7, \# B 145(ع)). These textures are in contrast to those obtained in the room temperature run as well as previous room temperature measurements. ${ }^{24-26}$

In order to interpret these textures generated at high temperature in hcp iron we turn to polycrystal plasticity modeling. Texture development depends on the deformation geometry as well as the relative activities of the different deformation modes such as slip or deformation twinning. A comparison of textures generated by polycrystal plasticity modeling to experimental textures can provide insight into which slip systems or twin modes are active under a given set of conditions. For these simulations we used the Los Alamos viscoplastic self-consistent code (VPSC). ${ }^{56,57}$ The VPSC model treats each grain as an inclusion in a homogeneous but anisotropic medium that has the average properties of the polycrystal. As deformation proceeds, crystals deform and rotate to generate preferred orientation. By applying different critical resolved shear stresses (CRSS) to slip systems and twin modes, the model will favor one deformation mode over another. This will result in different textures for different combinations of deformation modes. By determining which simulated texture most closely resembles the experimental texture, we can infer which slip systems and twining modes are active under the experimental conditions. Since the experimental $\varepsilon$ phase inherits a transformation texture prior to deformation, we used 2000 grains weighted to have a starting texture as obtained after recrystallization (Figure 7, \# B 138(e)). This was done in order to account for sample history, i.e. the initial development of a transformation texture followed by subsequent recrystallization. We apply an incremental deformation path of $20 \%$ compressive strain. Results are show in Figure 8 and values for critical resolved shear stresses activities of deformation modes are given in Table I.

Dominant basal slip produces a texture similar to that observed experimentally

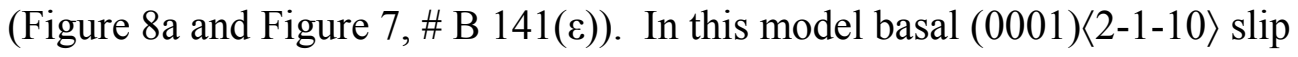
accommodates most of the deformation with moderate activity of prismatic $\{10-10\}<-12$ $10\rangle$ slip (Table I). Twinning modes are inactive in this model. This model accurately reproduces the girdle offset $30^{\circ}$ from 0001 and the maximum at 11-20. However, dominant basal slip generates a minimum at 01-10 that is not observed experimentally (Figure 8a and Figure 7, \# B 141(ع)). It is noted that for dominant basal slip, higher 
strains will cause the girdle $30^{\circ}$ offset from 0001 to converge at 0001 , as has been observed in room temperature compression of hcp Fe. ${ }^{24-26}$ Dominant pyramidal $\langle\mathrm{a}+\mathrm{c}\rangle\{2-$ $1-12\}\langle 2-1-13\rangle$ slip and basal (0001) $\langle 2-1-10\rangle$ slip produces a texture that is most similar to the experimental textures (Figure 8b and Figure 7, \# B 141(ع)). This model reproduces the girdle offset $30^{\circ}$ from 0001 as well as a broad maximum at 11-20 that extends about the periphery. Dislocations consistent with pyramidal $\langle a+c\rangle$ slip on $\{2-1-12\}\langle 2-1-13\rangle$ were observed with TEM in Ni-Cr stainless steel and it was suggested that this system may be active in $\varepsilon-\mathrm{Fe}^{27}$. Additionally it is expected that many slip systems should become active at elevated temperature as CRSSs become softer and more similar.

Next we are going to explore the influence of twinning. In hcp metals "tensile" twinning occurs if a crystal is extended along the c-axis and "compressive" twinning if it is compressed along the c-axis. Dominant tensile twining on $\{10-12\}\langle-1011\rangle$ will produce a broad maximum around 0001 (Figure $8 \mathrm{c}$ ). In this model twinning initially accommodates most of the deformation with the other deformation modes relatively inactive. As favorably oriented grains become twinned and tensile twinning is exhausted other deformation modes take over (Table I). At 20\% strain basal (0001) $\langle 2-1-10\rangle$ slip and pyramidal $\langle\mathrm{a}+\mathrm{c}\rangle\{2-1-12\}\langle 2-1-13\rangle$ slip accommodate most of the deformation (Table I). Dominant compressive twinning on $\{2-1-12\}\langle 2-1-1-3\rangle$, with moderate activity of other slip systems, will produce a girdle around the periphery of the IPF (Figure 8d). Again twinning initially accommodates most of the deformation, but at $20 \%$ strain prismatic and basal slip are dominant (Table I). For twinning simulations slip systems were identically weighted and differences in activities of auxiliary slip systems is due to maintaining compatibility for different twin modes. Compressive twinning could produce the girdle observed late in the experiment (Figure 7, \# B 145(ع)). However, mechanical twinning is generally a low temperature deformation mechanism that is active early in deformation ${ }^{58,59}$ and so is unlikely in this case.

While this new rDAC heating technique proved very successful, there is still room for improvement. The major short coming of this technique is the large temperature gradient within the sample. This may partly be overcome with better insulating material between the diamond anvils and the sample. The insulating material used here was polycrystal $\mathrm{MgO}$ powder which was often observed to flow away from the 
sample during heating. It is likely that single crystal $\mathrm{MgO}$ or sapphire may provide better insulation and more even heating. This problem may be less pronounced in oxides and silicates as these samples have low enough thermal conductivity that they can selfinsulate. Another approach that could help resolve the issue with temperature gradients is to defocus the laser to provide a larger hotspot size on the sample. The main drawback to this is that the amount of power needed to heat the sample is much greater than with a well focused laser beam and significant amounts of heat are absorbed by the body of the rDAC.

Another technique that is being developed for in-situ radial diffraction and high pressure and temperature deformation is a resistively heated rDAC. ${ }^{60}$ This device has the advantage over laser heating that samples have a much more homogeneous temperature. This technique however requires a much more complicated setup and is limited to temperatures less than $1400 \mathrm{~K}$. For temperatures greater than this laser heating remains the only option for in-situ radial diffraction experiments with deformation at simultaneous high-pressure and temperature.

\section{Conclusions}

A method is described for deformation experiments at ultra-high pressure $(>20$ $\mathrm{GPa}$ ) and temperature (>1900 K) using a DAC in radial diffraction geometry, combined with laser heating. Pressure/stress is remote controlled with a membrane system. This method is applied to iron, investigating texture changes during deformation and phase transformation in three phases in-situ: bcc $(\alpha)$, fcc $(\gamma)$ and hcp $(\varepsilon)$. Compression textures of bcc and fcc iron correspond to those described in the literature, establishing in situ rDAC experiments as a reliable method. For hcp Fe deformed at high-pressure and hightemperature different slip systems are active than at low-temperature. At hightemperature pyramidal $\langle a+c\rangle$ slip on $\{2-1-12\}\langle 2-1-13\rangle$ appears to be dominant while at room-temperature basal (0001) $\langle 2-1-10\rangle$ slip is dominant. The bec to hep Fe transformation appears to follow the Burgers' relationship with the additional observation that at room temperature grains oriented with $\{112\}$ at high angles to compression are more favored to transform to the $\varepsilon$-phase. The bcc to fcc Fe phase transformation follows the Kurdjumov-Sachs orientation relationship. For all transformations observed here, 
variant selection plays an important role with the exception of the back transformation from hcp Fe to bcc Fe at room temperature .

\section{Acknowledgements:}

The Advanced Light Source is supported by the Director, Office of Science, Office of Basic Energy Sciences, Materials Sciences Division, of the U.S. Department of Energy under Contract No. DE-AC02-05CH11231 at Lawrence Berkeley National Laboratory and University of California, Berkeley, California. COMPRES, the Consortium for Materials Properties Research in Earth Sciences under NSF Cooperative Agreement EAR 01-35554 supported this project through funding of MK, as well as crucial beamline-equipment. HRW and LM acknowledge support from CDAC and NSF (EAR 0337006). 


\section{Figure Captions}

Figure 1: P-T phase diagram for Fe. ${ }^{8}$ The $\alpha$-phase has a bcc structure, the $\gamma$-phase has an fcc structure, and the $\varepsilon$-phase is hcp. Black arrows show the path for the room temperature run $(\mathrm{A})$ and the high temperature run $(\mathrm{B})$.

Figure 2: "Exploded" view of the loading frame. A frame body houses the DAC while a base plate holds the membrane against the DAC piston. Inflating the gas membrane applies force to drive the piston. The cap of the loading frame has a window to allow imaging and laser access.

Figure 3: Picture of the loading frame and two rDACs. From left to right, top spacer for Merkel style panoramic rDAC, Merkel style panoramic rDAC, bottom spacer, and Mao type panoramic rDAC and gas membrane inside assembled loading frame.

Figure 4: Graph of pressure versus run number for experiment cycle 1, with IPFs for selected points shown. Compression of the bcc phase yields maxima at 001 and 111 which are attributed to slip on $\{110\}\langle 111\rangle$. Upon completion of the phase transformation, hcp Fe develops a maximum at 11-20 consistent with Burgers' relationship. During decompression, the hep phase develops an 0001 maximum. This is attributed to basal (0001) $\langle 2-1-10\rangle$ slip. Inset: Lines connecting the different phases show Burgers' relationship ${ }^{30}$ for the bcc (top) to hep (bottom) transformation. Bcc Fe orientations with $112_{\mathrm{bcc}}$ close to compression transform first generating a maximum at

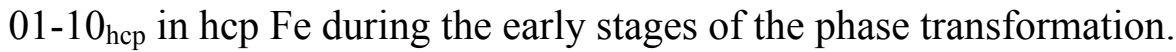

Figure 5: Graph of phase proportions versus run number for experiment cycle 1 at room temperature. At the start of the experiment the sample is pure bcc Fe. At pressures greater than $10 \mathrm{GPa}$ the hcp phase appears and becomes dominant. At $\sim 22 \mathrm{GPa}$ the sample has fully converted to hcp Fe. On decompression the sample reverts to the bcc phase 
Figure 6: "Unrolled" diffraction images from experiment cycle 2. a) start of experiment, b) just before laser heating, c) during heating at $1900 \mathrm{~K}$, and d) after compression to 30 GPa at $1800 \mathrm{~K}$. During heating both the fcc $(\gamma)$ and hcp $(\varepsilon)$ phases appear as well as a highly recrystallized region containing a second higher temperature fcc phase $\left.\left(\gamma_{2}\right) \mathrm{c}\right)$; with compression to $30 \mathrm{GPa}$ the fcc phase disappears and the hcp phase becomes dominant d). Major peaks are labeled and the compression and extension directions are shown with block arrows

Figure 7: Graph showing phase proportions vs run number for the bcc, fcc, and hcp phases with selected IPFs for experiment cycle 2. At the start of the experiment the sample is all bcc Fe. With heating to $1900 \mathrm{~K}$ we see the appearance of fcc and hcp Fe and with compression the bcc and fcc phases decrease in proportion and the hcp phase becomes dominant. With heating hep Fe develops a maximum at 01-10 (\# B 138(E)). This can be attributed to recrystallization switching 11-20 orientations to 01-10 ${ }^{55}$. With

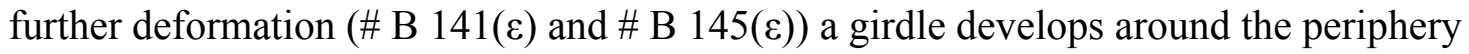
while the girdle $30^{\circ}$ offset from 0001 remains. Inset shows the Kurdjumov-Sachs orientation relation for bcc (top) to fcc (bottom) transformation ${ }^{52}$. Lines connecting the two phases show illustrate the equivalent orientations in the two phases.

Figure 8: VPSC results for a) dominant basal $(0001)\langle 2-1-10\rangle$ slip, b) dominant pyramidal $\langle\mathrm{a}+\mathrm{c}\rangle\{2-1-12\}\langle 2-1-13\rangle$ slip, c) dominant tensile $\{10-12\}\langle-1011\rangle$ twinning, and d) dominant compressive $\{2-1-12\}\langle 2-1-1-3\rangle$ twinning. Model b provides the closest result to the experimental data (see Table I for critical resolved shear stress and system activities). 


\section{Table and Caption}

Table I: Critical resolved shear stresses (CRSS) and activities (ACT) of the different deformation modes for model results shown in Figure 8. Activities are show for the start of the simulation and the end of the simulation.

\begin{tabular}{|c|c|c|c|c|c|c|c|c|c|c|c|c|c|c|c|c|c|c|}
\hline \multirow[b]{2}{*}{ Model } & \multicolumn{3}{|c|}{ Prismatic } & \multicolumn{3}{|c|}{ Basal } & \multicolumn{3}{|c|}{ Pyramidal $\langle a\rangle$} & \multicolumn{3}{|c|}{ Pyramidal $\langle\mathrm{a}+\mathrm{c}\rangle$} & \multicolumn{3}{|c|}{ Tensile twinning } & \multicolumn{3}{|c|}{$\begin{array}{l}\text { Compressive } \\
\text { twinning }\end{array}$} \\
\hline & CRSS & $\begin{array}{r}\mathrm{AC} \\
\text { start }\end{array}$ & $\begin{array}{l}\% \\
\text { end }\end{array}$ & CRSS & $\begin{array}{r}\mathrm{AC}^{\prime} \\
\text { start }\end{array}$ & $\begin{array}{l}\% \% \\
\text { end }\end{array}$ & CRSS & $\begin{array}{r}\mathrm{AC}^{\prime} \\
\text { start }\end{array}$ & $\begin{array}{l}\Gamma \% \\
\text { end }\end{array}$ & CRSS & $\begin{array}{r}\mathrm{AC}^{\prime} \\
\text { start }\end{array}$ & $\begin{array}{l}\Gamma \% \\
\text { end }\end{array}$ & CRSS & $\begin{array}{r}\mathrm{AC} \\
\text { start }\end{array}$ & $\begin{array}{l}\Gamma \% \\
\text { end }\end{array}$ & CRSS & $\begin{array}{r}\mathrm{AC} \\
\text { start }\end{array}$ & $\begin{array}{l}\Gamma \% \\
\text { end }\end{array}$ \\
\hline $\mathrm{A}$ & 2 & 24 & 20 & 1 & 47 & 49 & 3 & 12 & 10 & 4 & 18 & 21 & 3 & 0 & 0 & 3 & 0 & 0 \\
\hline B & 2 & 8 & 9 & 1 & 25 & 23 & 3 & 4 & 4 & 1 & 63 & 64 & 3 & 0 & 0 & 3 & 0 & 0 \\
\hline $\mathrm{C}$ & 2 & 19 & 10 & 2 & 27 & 40 & 3 & 13 & 10 & 4 & 11 & 38 & 1 & 31 & 2 & 3 & 0 & 1 \\
\hline $\mathrm{D}$ & 2 & 24 & 35 & 2 & 24 & 22 & 3 & 15 & 19 & 4 & 10 & 18 & 3 & 0 & 0 & 1 & 28 & 6 \\
\hline
\end{tabular}




\section{Supplemental Material}

Table SM-I: Experimental conditions from cycle 1 (room temperature). Associated errors are given in parenthesis. Texture strength is given by the texture index $\left(\mathrm{F}_{2}\right)$ which is a measure of texture sharpness.

Table SM-II: Experimental condition from cycle 2. Errors are given in parentheses and texture strength is given by the texture index $\left(\mathrm{F}_{2}\right)$ which is a measure of texture sharpness. 


\section{References}

${ }^{1}$ R.J. Hemley, H-K. Mao, G. Shen, J. Badro, P. Gillet, M. Hanfland, D. Häusermann, Science, 276, 316, 1242 (1997).

${ }^{2}$ A.K. Singh, C. Balasingh, H-K. Mao, R.J. Hemley, J.F. Shu, J. Appl. Phys. 83, 12, 7567 (1998).

${ }^{3}$ H.K. Mao, J. Shu, G. Shen, R.J. Hemley, B. Li, A.K. Singh, Nature 399, 280 (1999).

${ }^{4}$ H-R. Wenk, I. Lonardelli, S. Merkel, L. Miyagi, J. Pehl, S. Speziale, C.E. Tommaseo, J. Phys. Cond. Matter, 18, S933 (2006).

${ }^{5}$ M. Kunz, W.A. Caldwell, L. Miyagi, H-R. Wenk, Review of Scientific Instruments, 78 063907-1 (2007).

${ }^{6}$ R. LeToullec, J.P. Pinceaux, P. Loubeyre, High Pressure Research 1, 77 (1988).

${ }^{7}$ W.L. Mao H-K. Mao, J. Phys. Cond. Matter, 18, S1069 (2006).

${ }^{8}$ G. Shen, H-K. Mao, R.J. Hemley, T.S. Duffy, M.L. Rivers, Geophys. Res. Lett. 25, 373 (1998).

${ }^{9}$ M.H. Manghnani, L.C. Ming, N. Nakagiri, in High-Pressure Research in Mineral Physics, Geophys. Monogr. Ser., vol. 39, edited by M.H. Manghnani and Y. Syono [AGU, Washington, D. C., 1987], pp. 155-163.

${ }^{10}$ E. Huang, W.A. Basset, P. Tao, in High-Pressure Research in Mineral Physics, Geophys. Monogr. Ser., vol. 39, edited by M.H. Manghnani and Y. Syono [AGU, Washington, D. C., 1987], pp. 165-172.

${ }^{11}$ N. von Bargen, R. Boehler, High Pressure Res. 6, 133 (1990).

${ }^{12}$ S. Akimoto, T. Suzuki, T. Yagi, O. Shimomura, in High-Pressure Research in Mineral Physics, Geophys. Monogr. Ser., vol. 39, edited by M.H. Manghnani and Y. Syono [AGU, Washington, D. C., 1987], pp. 149-154.

${ }^{13}$ H-K. Mao, P.M. Bell, C. Hadidiacos, in High-Pressure Research in Mineral Physics, Geophys. Monogr. Ser., vol. 39, edited by M.H. Manghnani and Y. Syono [AGU, Washington, D. C., 1987], pp. 135-138.

${ }^{14}$ R. Boehler, M. Nicol, M.L. Johnson, in High-Pressure Research in Mineral Physics, Geophys. Monogr. Ser., vol. 39, edited by M.H. Manghnani and Y. Syono [AGU, Washington, D. C., 1987], pp. 173-176. 
${ }^{15}$ R.J. Hemley, H-K. Mao, Int. Geol. Rev. 43, 1 (2001).

${ }^{16}$ Y. Ma, M. Somayazulu, G. Shen, H-K. Mao, J. Shu, R.J. Hemley, Phys. of The Earth Planet. Inter., 143-144, 455 (2004).

${ }^{17}$ G. Poupinet, R. Pillet, A. Souriau, Nature 305, 204 (1983).

${ }^{18}$ A. Morelli, A.M. Dziewónski, J.H. Woodhouse, Geophys. Res. Lett. 13, 1545 (1986).

${ }^{19}$ H-R. Wenk, T. Takeshita, R. Jeanloz, G.C. Johnson, Geophys. Res. Lett. 15, 76 (1988).

${ }^{20}$ S. Yoshida, I. Sumita, M. Kumazawa, J. Geophys. Res. 101, 28085 (1996).

${ }^{21}$ S. Karato, Nature 402, 871-873 (1999).

${ }^{22}$ H-R. Wenk, J.R. Bamgardner, R.A. Lebensohn, C.N. Tomé, J. Geophys. Res. 105, 5663 (2000a).

${ }^{23}$ B.A. Buffet, H-R. Wenk, Nature 413, 60 (2001).

${ }^{24}$ H-R. Wenk, S. Matthies, R.J. Hemley, H-K. Mao, J. Shu, Nature 405, 1044 (2000b).

${ }^{25}$ S. Merkel, H-R. Wenk, P. Gillet, H-K. Mao, R.J. Hemley, Phys. Earth Planet.Inter., 145, 239 (2004).

${ }^{26}$ W.L. Mao, V.V. Struzhkin, A. Baron, S. Tsutsui, C. Tommaseo, H-R. Wenk, M. Hu, P. Chow, W. Sturhahn, J. Shu, R.J. Hemley, D.L. Heinz, H-K. Mao, JGR (in press), (2008).

${ }^{27}$ J.P. Poirier, F. Langenhorst, Phys. Earth Panet. Int. 129, 347 (2002).

${ }^{28}$ C. Barrett, T.B. Massalski, Structure of Metals (Pergamon Press, Oxford, UK, 1980).

${ }^{29}$ A.D. Rollett, S.I. Wright, in Texture and Anisotropy. Preferred orientations in Polycrystals and their Effect on Materials Properties edited by U.F. Kocks, C.N. Tomé, H-R. Wenk, [Cambridge University Press, Cambridge, 2000] Ch. 5, pp. 179-239.

${ }^{30}$ W.G. Burgers, Physica 1, 561 (1934).

${ }^{31}$ M. Kunz, A.A. MacDowell, W.A. Caldwell, D. Cambie, R.S. Celestre, E.E. Domning, R.M. Duarte, A.E. Gleason, J.M. Glossinger, N. Kelez, D.W. Plate, T. Yu, J.M. Zaug, H.A. Padmore, R. Jeanloz, A.P. Alivisatos, S.M. Clark, Journal of Synchrotron Radiation, 12, 650 (2005). 
${ }^{32}$ W.A. Caldwell, M. Kunz, R.S. Celestre, E.E. Domning, M.J. Walter, D. Walker, J. Glossinger, A.A. MacDowell, H.A. Padmore, R. Jeanloz, S.M. Clark, Nuclear Instruments and Methods in Physics research A, 582, 221 (2007).

${ }^{33}$ S. Merkel, T. Yagi, Review of Scientific Instruments 76, 046109-3 (2005).

${ }^{34}$ J. Zhang, F. Guyot, Physics and Chemistry of Minerals 26, 206 (1999).

${ }^{35}$ H-K. Mao, Y. Wu, L.C. Chen, J.F. Shu, A.P. Jephcoat, J. Geophys. Res., 95, 737-21 (1990).

${ }^{36}$ Y.W. Fei, A. Ricolleau, M. Frank, K. Mibe, G.Y. Shen, V. Prakapenka, PNAS 104, 9182 (2007).

${ }^{37}$ L. Lutterotti, S. Matthies, H-R. Wenk, Int. U. Crystallogr. Comm. Powder Diffr. Newslett. 21, 14 (1999).

${ }^{38}$ S. Matthies, G.W. Vinel, Physica Status Solidi B-Basic Research 112, K115 (1982).

${ }^{39}$ H-R. Wenk, S. Matthies, J. Donovan, D. Chateigner, Journal of Applied Crystallography 31, 262 (1998).

${ }^{40}$ N.R. Barton, D.J. Benson, R. Becker, Modelling and Simulation in Materials Science and Engineering, 13, 707 (2005).

${ }^{41}$ N. Hamaya, S. Akimoto, High Temp. High Pressures, 13, 347 (1981).

${ }^{42}$ F.M. Wang, R. Ingalls, Phys. Rev. B, 57, 5647 (1998).

${ }^{43}$ Z. Nishiyama, Martensitic transformation. (New York, Academic Press, 1978).

${ }^{44}$ J.R. Morris, K.M. Ho, Phys. Rev. B, 63, 224116 (2001).

${ }^{45}$ J.A. Krumhansl, Phase Transitions 65,109 (1998).

${ }^{46} \mathrm{Yu}$ N. Gornostyrev, M.I. Katsnelson, A.R. Kuznetsov, A.V. Trefilov, JETP Lett. 70, 380 (1999).

${ }^{47}$ A.R. Kuznetsov, Yu N. Gornostyrev, M.I. Katsnelson, A.V. Trefilov, Materials Science and Engineering A 309-310, 168 (2001).

${ }^{48}$ H-R. Wenk, G. Canova, Y. Brechet, L. Flandin, Acta Materialia 45, 3283 (1997).

${ }^{49}$ H-R. Wenk, I. Lonardelli, D. Williams, Acta Materialia 52, 1899 (2004).

${ }^{50}$ A.P. Hammersley, ESRF Internal Report, ESRF97HA02T (1997). 
${ }^{51}$ R.K. Ray, J.J. Jonas, M.P. Butrón-Guillén, J. Savoie, ISIJ International 34, 927 (1994).

${ }^{52}$ G. Kurdjumov, G. Sachs, Zeitschrift für Physik A Hadrons and Nuclei 64, 325 (1930).

${ }^{53}$ H-R. Wenk, I. Huensche, L. Kestens, Metallurgical and Materials Transactions A 38, 261 (2007).

${ }^{54}$ S. Speziale, I. Lonardelli, L. Miyagi, J. Pehl, C.E. Tommaseo, H-R. Wenk, J. Phys. Cond. Matter 18, S1007 (2006).

${ }^{55}$ I. Lonardelli, N. Gey, H-R. Wenk, L. Lutterotti, S. Vogel, M. Humbert, Acta Mater. 55, 5718 (2007).

${ }^{56}$ R.A. Lebensohn, C.N. Tomé, Mater. Sci. Eng. A175, 71 (1994).

${ }^{57}$ C.N. Tomé, G.R. Canova, Self-consistent modeling of heterogeneous plasticity. in Texture and Anisotropy. Preferred orientations in Polycrystals and their Effect on Materials Properties edited by U.F. Kocks, C.N. Tomé, H-R. Wenk, [Cambridge University Press, Cambridge, 2000] Ch. 11, pp. 466-510.

${ }^{58}$ C.N. Tomé, S.R. Agnew, W.R. Blumenthal, M.A.M. Bourke, D.W. Brown, G.C. Kaschner, P. Rangaswamy, Mat. Sci. For., 408-412, 263 (2002).

${ }^{59}$ L. Wu, A. Jain. D.W. Brown, G.M. Stoica, S.R. Agnew, B. Clausen, D.E. Fielden, P.K. Liaw, Acta Materialia 56, 688 (2008).

${ }^{60}$ H-P. Liermann, S. Merkel, L. Miyagi, H-R. Wenk, G. Shen, H. Cynn, W.J. Evans, Eos Trans. AGU 88(52), Fall Meet. Suppl., Abstract MR43A-0975 (2007). 


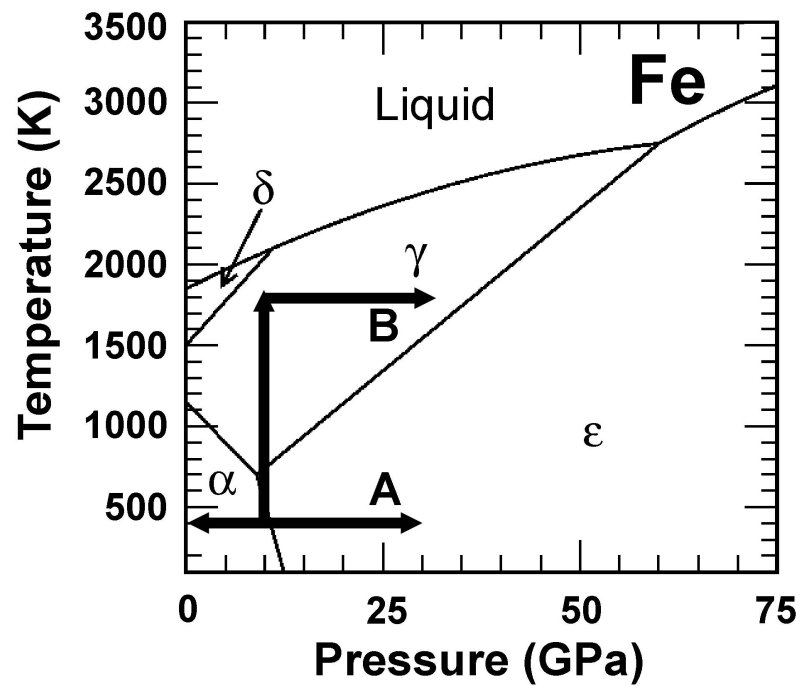




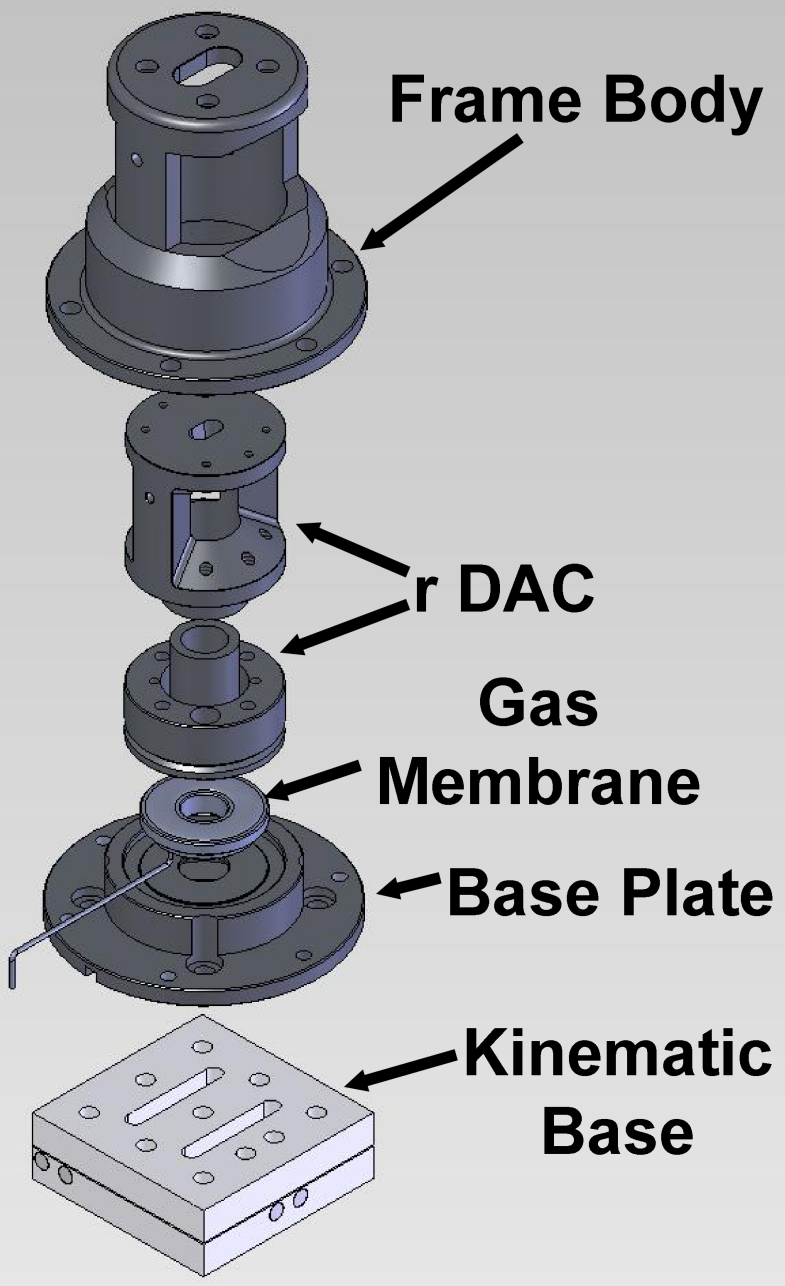




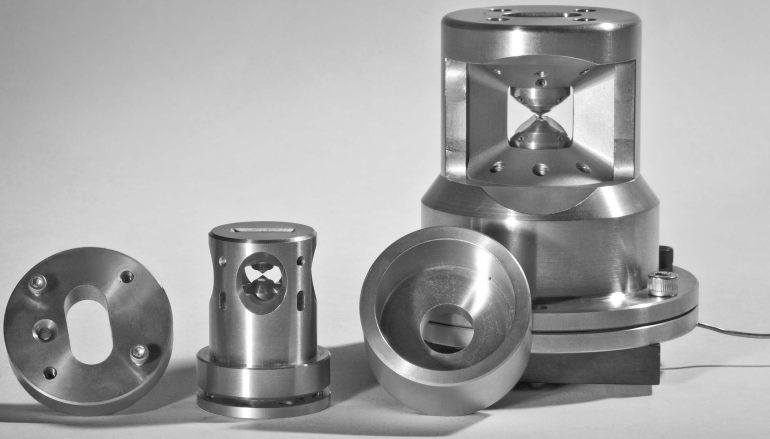


Cycle 1

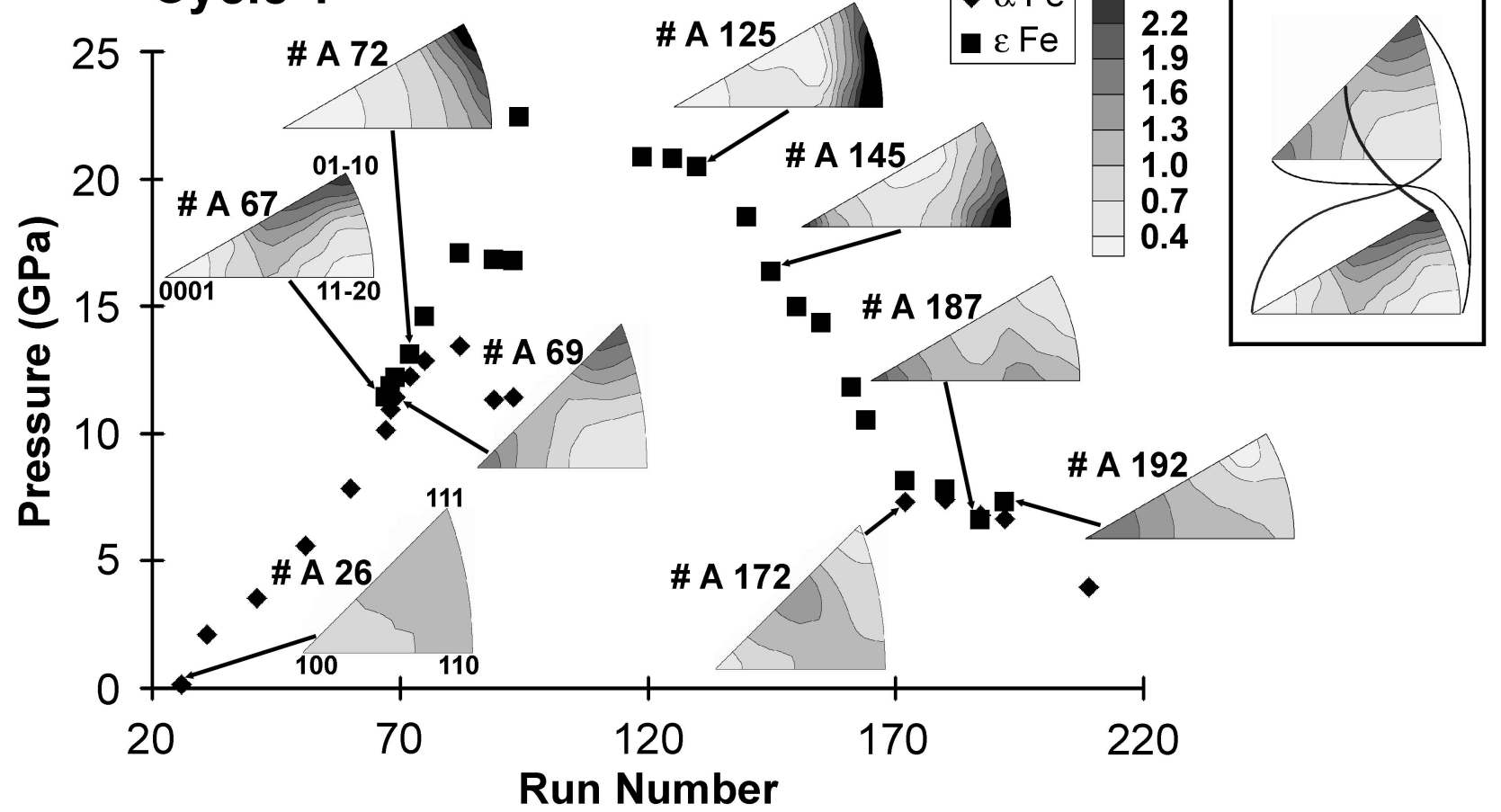




\section{Cycle 1}

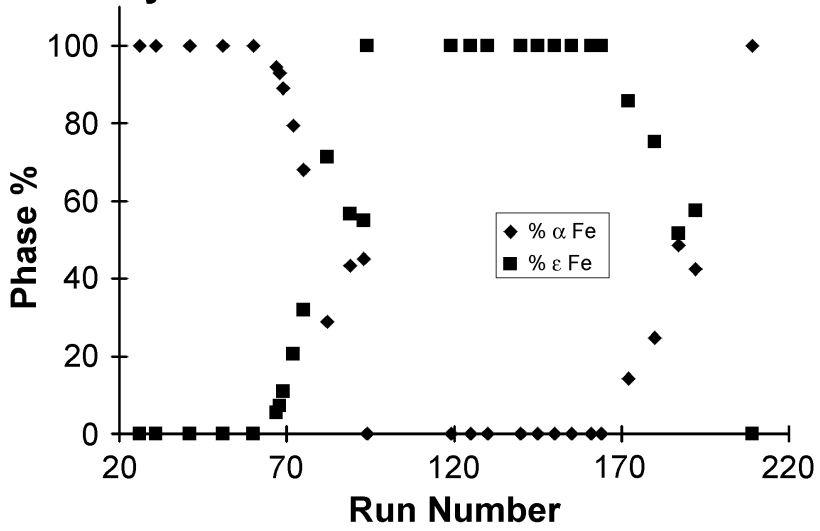


a) \# B 115

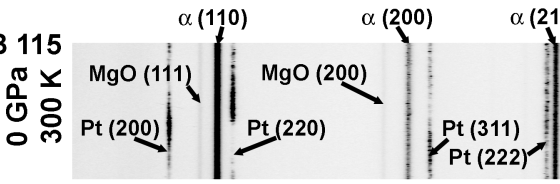

$\alpha(200)$

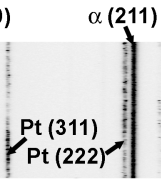

b) \# B 126

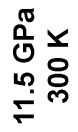

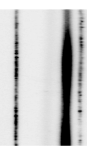

$$
\alpha(110)
$$
$\mathrm{MgO}(200)$ $\mathrm{Pt}(220)$

C) \# B 133
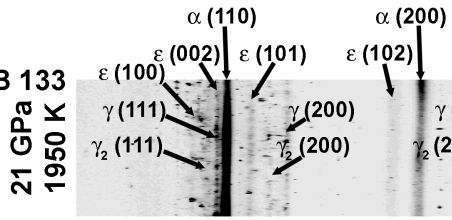

(101) $\varepsilon(102)$

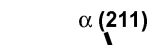

Pt (400)

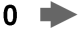
90

$90<$ 
Cycle 2

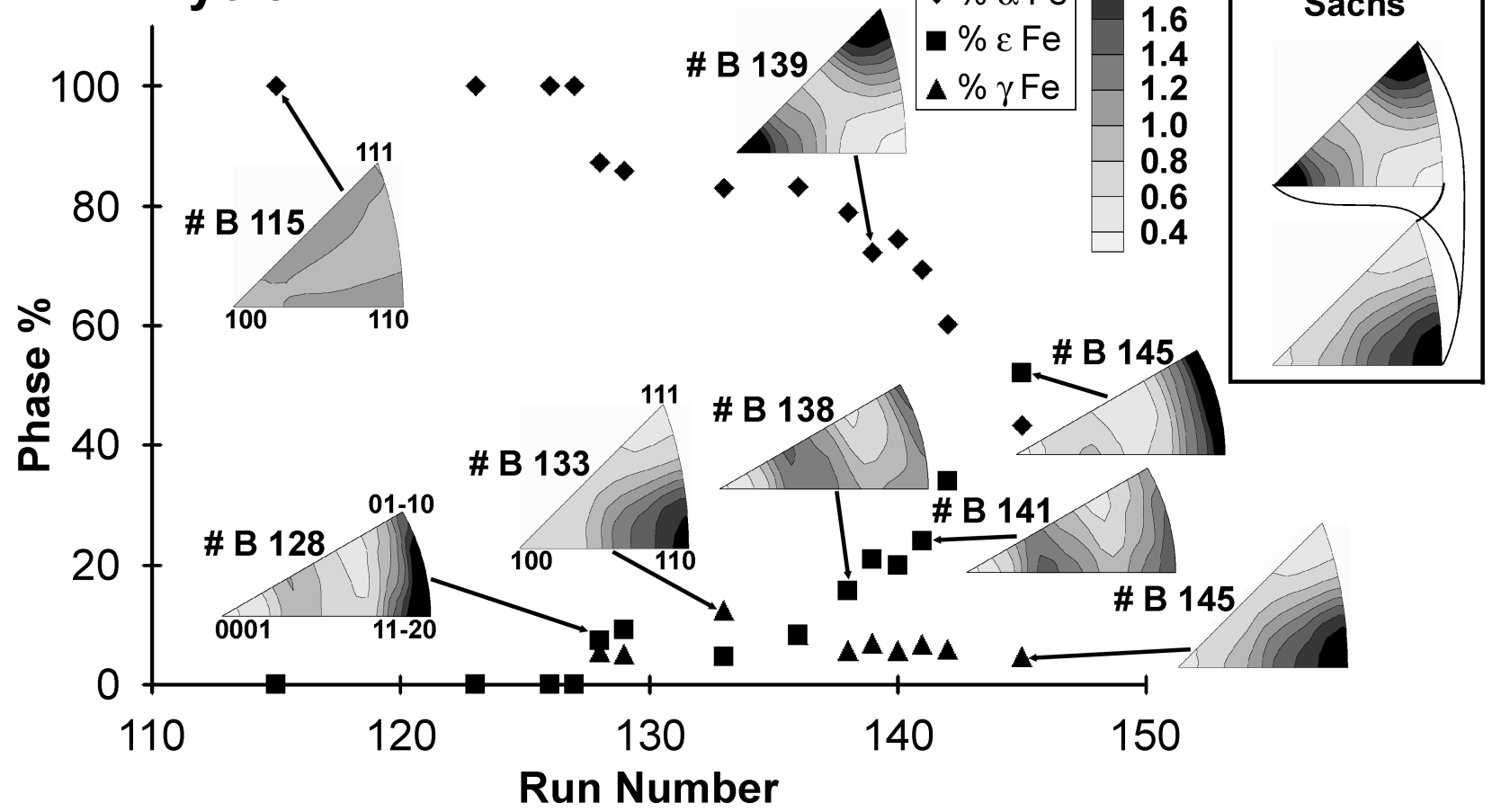


a) $\quad 01-10$ b)

0001

11-20 c)

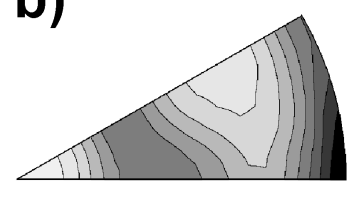

d)
1.8

1.6

1.4

1.2

1.0

0.8

0.6

0.4 